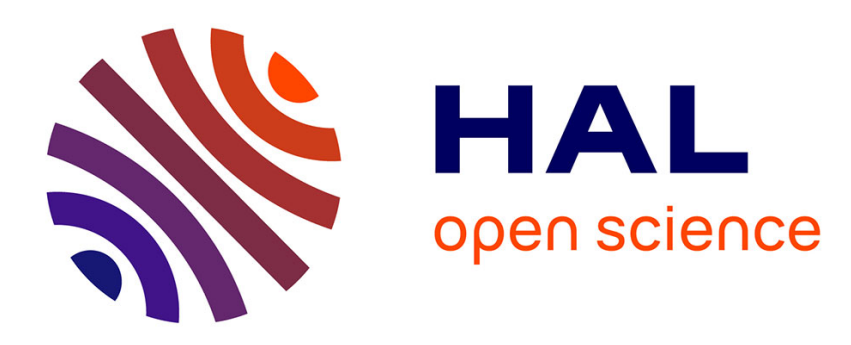

\title{
Indirect boundary force measurements in beam-like structures using a derivative estimator Simon Chesné
}

\section{To cite this version:}

Simon Chesné. Indirect boundary force measurements in beam-like structures using a derivative estimator. Journal of Sound and Vibration, 2014, 333 (24), pp.6438-6452. 10.1016/j.jsv.2014.07.026 . hal-01070547

\section{HAL Id: hal-01070547 \\ https://hal.science/hal-01070547}

Submitted on 29 Jun 2016

HAL is a multi-disciplinary open access archive for the deposit and dissemination of scientific research documents, whether they are published or not. The documents may come from teaching and research institutions in France or abroad, or from public or private research centers.
L'archive ouverte pluridisciplinaire HAL, est destinée au dépôt et à la diffusion de documents scientifiques de niveau recherche, publiés ou non, émanant des établissements d'enseignement et de recherche français ou étrangers, des laboratoires publics ou privés. 


\title{
Indirect boundary force measurements in beam-like structures using a derivative estimator
}

\author{
Simon CHESNE \\ LaMCoS, INSA-Lyon, CNRS UMR5259, \\ 18, rue des Sciences, F69621 Villeurbanne, France \\ Author Manuscript, Published in Journal of Sound and Vibration, \\ Vol. 333(24), 2014
}

\begin{abstract}
This paper proposes a new method for the identification of boundary forces (shear force or bending moment) in a beam, based on displacement measurements. The problem is considered in terms of the determination of the boundary spatial derivatives of transverse displacements. By assuming the displacement fields to be approximated by Taylor expansions in a domain close to the boundaries, the spatial derivatives can be estimated using specific point-wise derivative estimators. This approach makes it possible to extract the derivatives using a weighted spatial integration of the displacement field. Following the theoretical description, numerical simulations made with exact and noisy data are used to determine the relationship between the size of the integration domain and the wavelength of the vibrations. The simulations also highlight the self-regularization of the technique. Experimental measurements demonstrate the feasibility and accuracy of the proposed method.
\end{abstract}

Key-words:Numerical differentiation, Boundary identification, Indirect Force measurement

\section{Introduction}

Source characterization is a major issue in the analysis of structural vibrations. In many vibro-acoustic problems, structures are excited by their boundaries. The identification of boundary forces (such as shear forces or bending moments in beam-like structures) is not straightforward, because these physical quantities are not readily accessible and sensors can rarely be positioned at suitable locations. From a more general point of view, the 
identification of these forces/sources provides information related to the type of vibration generated. They make it possible to: analyse stress inside the structure, describe how vibration energy propagates, through the use of structural intensity calculations [1], [2], and localize external forces applied to the structure.

Force localisation and estimation is a wide subject. A lot approaches exist. These approaches include transfer-function-based methods [3], numerical model-based methods [4], modal analysis [5], methods using discretised differential motion equation [6], [7], [8], [9], or using an operator obtained with the Finite Element Method [10], etc... An interesting review of certain methods is proposed in [11]. Even if many force identification methods exist, few allow to identify boundary forces. Indeed, methods using discretized differential motion equation, which are very successful in a lot of applications, cannot be applied at structure boundaries. This is due to the fact that it is difficult to approximate the derivatives of a quantity, when this quantity is defined on only one side of a point of interest. The techniques described above are therefore unsatisfactory. The use of the annihilator operator, as proposed in this work, avoids this drawback during the derivative estimation step, which is a necessary step for this kind of approach. Other approaches as transfer-function-based [3] or transmissibility-function-based methods [12] work well if a measurement is located at the point where the force has to be estimated. This can be an important drawback. Indeed, the displacements measured at a structure's boundaries contain the highest levels of error, because the structure's absolute displacements are often very small at these locations, i.e. the signal-to-noise ratio is relatively low. Measurement errors in the vicinity of a structure's boundaries generally lead to large variations in the estimation of the boundary forces.

The goal of the technique proposed in the present study is to identify shear forces and/or bending moments at any point in a structure, in particular at its edges, where such forces can in some cases be associated with the sources. Few force identification methods focus on the boundaries. In the literature, boundary condition identification is a topic that is usually treated independently to sources identification.

Many methods for boundary conditions identification use Finite Element Approach. The general procedure is to apply idealized constraints or to make use of previous experience in modelling apparently similar boundaries. Boundary conditions may be determined by using conventional identification or updating methods [13]. In many cases, for this kind of inverse problems, measurement errors and ill-conditioning lead to unsatisfactory estimates of the spatial model by the equation error method [14], [15], [16]. Indeed measurement errors at the boundaries produce large variations in estimates of boundary parameters. One of the most popular methods in the determination of boundary parameters is the sensitivity method [17]; 
the difference between model predictions and test observations is defined using linearised first order sensitivities. Another approach [18] considers a method for the identification of structural boundary conditions which relies on the measured natural frequencies of the structure. From an another point of view, identification of boundary condition can be linked to the estimation of the boundary spatial derivatives.

If the spatial derivatives of the structural displacements are known, they can be used to determine these shear forces and/or bending moments. One derivative approximation technique involves the use of finite difference schemes [6], [8], spatial Fourier transforms [7], and modal expansions [19]. The major difficulty with this type of approach is that derivation amplifies the influence of noise in the recorded data, and the higher the order of the derivative, the greater the sensitivity of spatial derivatives to measurement errors. It is thus not possible to use such techniques, since they are, and must include, regularization developments, such as wave number filtering [8], modal truncation [19], regularization techniques [10] which require the adjustment of regularization parameters. To estimate derivatives, some approaches present a natural regularizing aspect using an integral formulation for boundary characterization [20][21][22][23], using polynomial approximation for damage detection [24], via operational calculus allowing algebraic derivative [23][25][26] or based on a least square approximation [27][28]. This kind of differentiation by integration methods allows an accurate and robust estimation of the derivative. The approach developed for temporal signals in [23] is used and adapted in the method detailed in this paper. Since boundary forces are proportional to the spatial derivatives of the structure's displacements, these approaches can be helpful to estimate these forces. But their quantification remains highly sensitive to uncertainties, especially at the boundaries, when post-processing techniques are applied.

To summarise, as for the force identification methods, two major difficulties are encountered when determining these spatial derivatives: they are highly sensitive to measurement uncertainties, and are difficult to measure at the structure's boundaries. The boundaries are often the transmission routes for vibrations, and measurements made at these points are also a major issue in terms of source identification.

It is known that classical characterization methods which use finite difference techniques are not suitable at boundaries. In [20], the spatial derivatives of a beam were determined using a weak form of the equation of motion. The principle consists in multiplying the equation of motion by a specific test function, and then integrating the resulting product by means of several successive integrations by parts. This approach allows the shear force (third spatial derivative) and/or the bending moment (second spatial derivative) to be extracted at one boundary of the integration 
domain. The integration is carried out using discretization, in which the displacements are measured with classical point sensors such as accelerometers, laser vibrometers, etc. The main drawback of this technique is the need to measure a spatial domain, which contains at least one wavelength at the angular frequency under consideration. An extension of this technique to the analysis of a plate structure presenting the same characteristics has also been proposed [21]. This approach was recently implemented directly, through the use of a distributed PVDF sensor. Since the output of a piezoelectric sensor corresponds to the weighted average of the surface strains in the region covered by the sensor electrodes, discrete integral calculations are not needed [22].

The method presented in the present paper is based on a numerical differentiation technique proposed by Mboup [23], which was initially developed to handle the case of temporal signals involving a very large number of data-points. This approach has been adapted to the case of structural vibrations, and modified to allow the derivatives to be estimated through the use of iterative integrals of the spatial displacement. The resulting estimation of a structure's spatial derivatives allows its boundary forces to be identified. This novel approach to the physics of the problem has an impact on the method's accuracy. Various limitations, such as the relationship between the size of the integration domain, the vibration wavelengths, sensitivity to noise, and the order of the Taylor expansion on which the approach is based, are identified. The first section of this paper describes the theory of the proposed method. The second section makes use of numerical simulations to evaluate its advantages and drawbacks. The last section describes the experimental validation of this technique, in the case of boundary shear force identification.

\section{Theory}

\subsection{System equations}

In this paper, the transverse vibrations of a beam are modelled using the standard Euler-Bernoulli theory for flexural motion. Harmonic oscillations are considered:

$$
v(x, t)=v(x) e^{j \omega t}
$$

where $v(x)$ is the transverse displacement and $\omega$ is the angular frequency. For the sake of clarity, the time dependence $e^{j \omega t}$ is simplified in the following. The equation of motion can thus be written as follows:

$$
E I \frac{\partial^{4} v}{\partial x^{4}}(x)-\rho S \omega^{2} v(x)=F(x)
$$

where $E$ is the complex Young's modulus, $I$ is the flexural inertia, $\rho$ is the mass density, $S$ is the cross-section of the beam and $F(x)$ is the excitation. 
The boundary forces identified in the present study can be computed using the spatial derivatives of $v(x)$ :

$$
\left\{\begin{array}{l}
T(x)=E I \frac{\partial^{3} v(x)}{\partial x^{3}} \\
M(x)=E I \frac{\partial^{2} v(x)}{\partial x^{2}}
\end{array}\right.
$$

where $T(x)$ is the shear force and $M(x)$ is the bending moment.

\subsection{Estimation of the boundary forces}

\subsubsection{Exact displacements}

It is known from beam theory [29] that transverse displacements verify Eq.(2) and can be written as the sum of two propagative and two evanescent bending waves on the left and right sides of the excitation point:

$$
\begin{aligned}
& v_{1}(x)=A_{1} \cos (k x)+B_{1} \sin (k x)+C_{1} e^{-k x}+D_{1} e^{k x} \\
& v_{2}(x)=A_{2} \cos (k x)+B_{2} \sin (k x)+C_{2} e^{-k x}+D_{2} e^{k x}
\end{aligned}
$$

where $v(x)=v_{1}(x)$ for $\left.\left.x \in\right] 0, x_{f}\right], v(x)=v_{2}(x)$ for $x \in\left[x_{f}, L[), k\right.$ is the natural wave number, satisfying the dispersion equation:

$$
k^{4}=\frac{\rho S}{E I} \omega^{2}
$$

and $A_{1}, B_{1}, C_{1}, D_{1}, A_{2}, B_{2}, C_{2}$, and $D_{2}$ are the wave amplitudes. These must be computed in order to satisfy boundary and continuity conditions at $x=0, x=x_{f}$ and $x=L$.

\subsubsection{Displacement approximation}

The first step in this approach is to consider that in a domain $\Omega=\left[0, x_{\Omega}\right]$ (with $x_{\Omega}<x_{f}$ ), the exact solutions (4) can be approximated by a truncation of the Taylor series expansion of the displacements. Assuming $v(x)$ to be an analytic function over $\Omega$, it can be approximated by:

$$
v(x) \simeq \sum_{i=0}^{N} \frac{\partial^{i} v}{\partial x^{i}}(0) \frac{x^{i}}{i !}
$$

where $N$ is the truncation number. Fig.1 illustrates this notion and its limitations for the first flexural mode of a simply supported beam, with $N=3, N=5$ and $N=7$. For the purposes of simplicity, the modes are normalized such that the maximum amplitude is equal to 1 . In the following examples, the proposed method is presented using these 3 truncation values, thereby illustrating the dependence of the computed approximation on this parameter. 


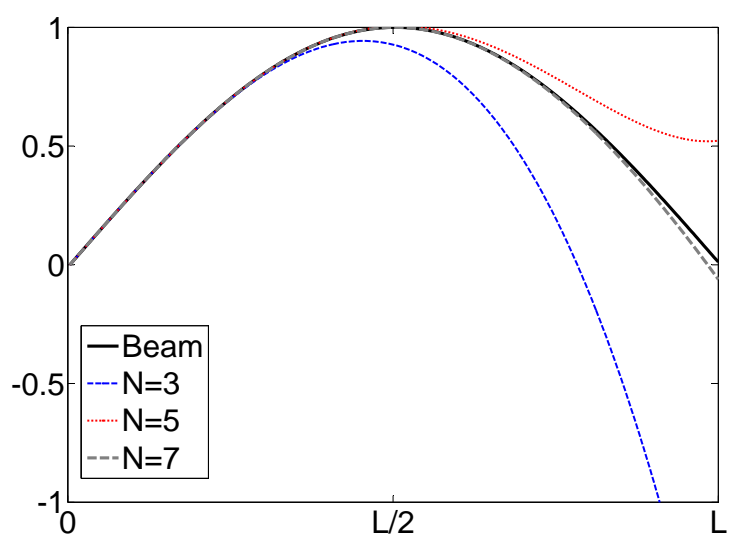

Figure 1: Taylor series approximation for beam displacement, at $\mathrm{x}=0$, for the first mode of a simply supported beam: $\omega=(\pi L)^{2} \sqrt{E I / \rho S}$

This figure contains various types of information. As could be expected, the greater the value of $N$, the more accurate the approximation. The approximation can nevertheless be accurate, whatever the truncation numbers is, if a small domain close to the boundary is considered. Although $\Omega$ includes the whole beam in this example, in practice this is not a good choice: it can be difficult to measure physical parameters over the whole structure, and at higher frequencies the Taylor approximation is clearly inaccurate (even with $N=7$ ), as shown in Fig. 2 for the $2^{\text {nd }}$ vibration mode.

It has been shown in previous studies $([20])$ that with this type of integral technique, the key parameter is not simply the angular frequency or the size of the domain $\Omega$, but rather the number of wavelengths contained in the domain $\Omega$.

Since a smaller number of wavelengths is present in $\Omega$ when the size of this domain is reduced, the accuracy of the Taylor series approximation is improved. The most well-adapted representations use the number of wavelengths included in $\Omega$, as shown Fig. 3, which do not depend on the size of $\Omega$, nor on $\omega$ (the angular frequency of the excitation): under such circumstances, the accuracy of the identification is related directly to the number of wavelengths present in the domain $\Omega$.

Fig. 4 illustrates the difference between the signal and its truncated Taylor expansion, in a domain limited to one half wavelength. The Taylor series approximation can be seen to deviate from the exact solution at $\lambda / 4$ when $N=3$, at $3 \lambda / 8$ when $N=5$, and at $\lambda / 2$ when $N=7$. It illustrates also the interest to use the number of wavelength in the considered domain 


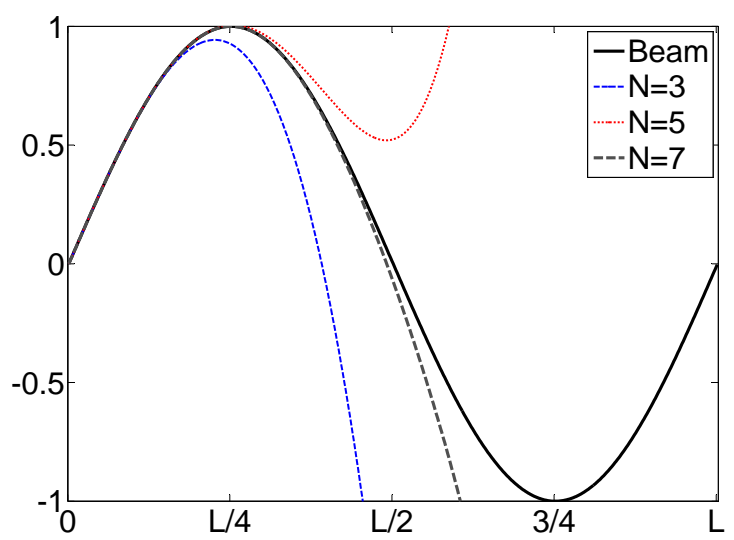

Figure 2: Taylor series approximation for beam displacements at $\mathrm{x}=0$, for the second mode of a simply supported beam: $\omega=(2 \pi L)^{2} \sqrt{E I / \rho S}$

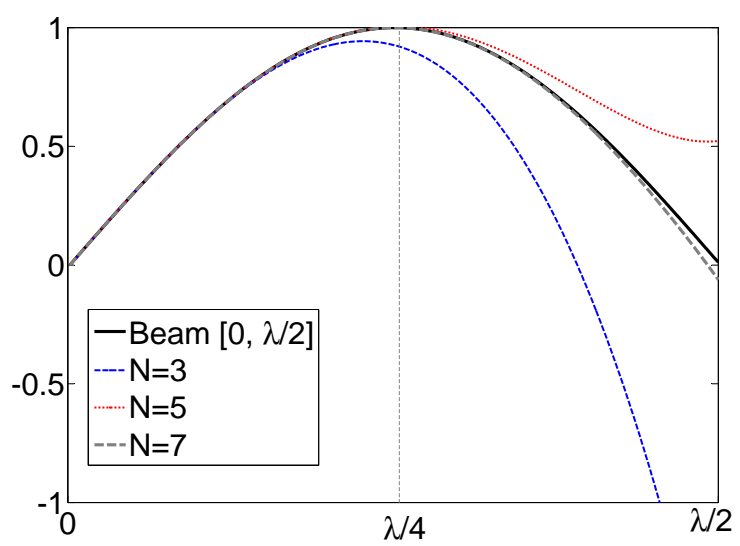

Figure 3: Taylor series approximation for $x=0, \Omega=[0 ; \lambda / 2], \forall \omega$

to plot the results. Indeed the Figs. 3 or 4 can correspond to the whole beam at its first natural frequency (Fig.1) or to half of the beam at its second natural frequency (Fig.2).

\subsubsection{Derivative estimation}

It has been shown that, through the use of an algebraic approach, a Taylor series expansion (6) can be used to numerically differentiate a quantity of 


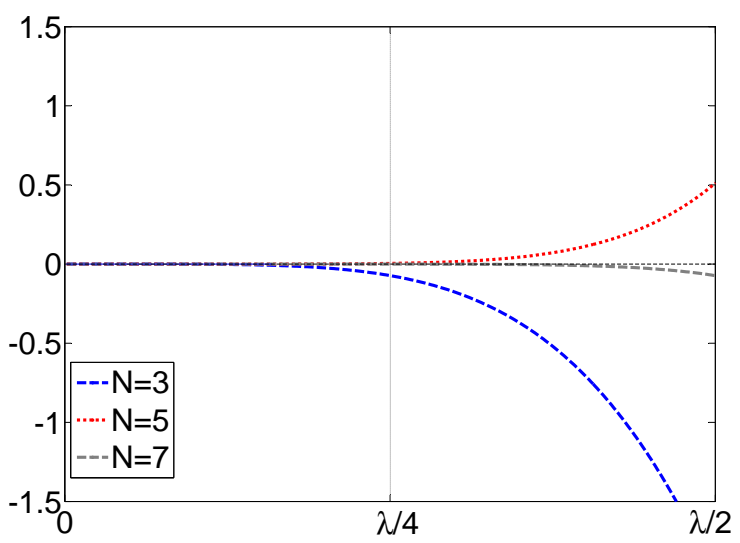

Figure 4: Error in the Taylor approximation for $x=0, \Omega=[0 ; \lambda / 2], \forall \omega$

interest [23]. This process can be summarised as follows:

In the Laplace domain, (6) can be written:

$$
v_{N}(s)=v(0) / s+v^{(1)}(0) / s^{2}+\ldots+v^{(N)}(0) / s^{N}
$$

where $v^{(i)}(x)$ is the $i^{t h}$ derivative of the beam's displacement at the coordinate $x$. In order to identify the boundary shear force, the third spatial derivative at $x=0$, which is $v^{(3)}(0)$ in the Laplace domain, needs to be estimated. All of the terms $v^{(p)}(0)$ in the expansion, for which $p \neq 3$, should thus be rejected. This can be achieved by applying various derivations or multiplications with the Laplace variable $s$. This operator is referred to as the 'annihilator' [23]. With standard finite element methods, an EulerBernoulli beam element is described by a third order polynomial function. In order to be coherent with the represented physical phenomenon, and to be able to compute the function's third derivative, a truncation number $N \geq 3$ has to be chosen.

This method is illustrated in the following example, using $N=3$. If both sides of expression (7) are multiplied by the annihilator $\Pi$, defined as:

$$
\Pi=s^{6} \frac{\partial}{\partial s} s^{2} \frac{\partial}{\partial s} s^{2} \frac{\partial}{\partial s} s,
$$

(7) then becomes:

$$
\begin{array}{r}
6 s^{-4} v(s)+18 s^{-3} v^{(1)}(s)+9 s^{-2} v^{(2)}(s)+s^{-1} v^{(3)}(s) \\
=-6 s^{-8} v^{(3)}(0)
\end{array}
$$

This can be re-written in the spatial domain as: 


$$
\begin{aligned}
6 \int_{\Omega}^{(4)} v(x) d x^{4}- & 18 \int_{\Omega}^{(3)} x v(x) d x^{3}+9 \int_{\Omega}^{(2)} x^{2} v(x) d x^{2} \\
& -\int_{\Omega}^{(1)} x^{3} v(x) d x=-6 A^{7} /(7 !) \frac{\partial^{3} v}{\partial x^{3}}(0)
\end{aligned}
$$

where $A$ is the length of the integration domain, defined by $\Omega=[0 ; A]$, and $\int^{(\alpha)}$ is the iterated integral of order $\alpha$.

The Cauchy formula can be used to transform this iterated integral into a simple one, giving:

$$
\int_{\Omega}^{(\alpha)}(-1)^{\beta} x^{\beta} v(x) d x^{\alpha}=\frac{1}{(\alpha-1) !} \int_{0}^{A}(A-x)^{\alpha-1}(-1)^{\beta} x^{\beta} v(x) d x
$$

Expression (10) can thus be written as:

$$
\frac{\partial^{3} v}{\partial x^{3}}(0)=\frac{-7 !}{6 A^{7}} \int_{0}^{A}\left[(A-x)^{3}-9(A-x)^{2} x+9(A-x) x^{2}-x^{3}\right] v(x) d x
$$

The integral expression (12) can be used to estimate the third derivative of the displacements, and has the substantial advantage of being based on integral calculations, thus making it potentially less sensitive to measurement noise.

As previously shown, when the truncation number $N$ used for the Taylor approximation is increased, the accuracy of the approximation $v_{N}(x)$ is improved. The third derivative can by computed using a longer Taylor expansion, and $\frac{\partial^{3} v_{N}}{\partial x^{3}}(0)$ can be computed as:

$$
\frac{\partial^{3} v_{N}}{\partial x^{3}}(0)=\int_{0}^{A} P_{N}(x) v(x) d x
$$

where $P_{N}(x)$ are the polynomial functions given in Appendix A, for $N=3$, 5 and 7 .

\subsection{Analytical results}

Three main sources of error are naturally present in this method:

- the influence of the truncation number $N$ corresponding to the displacement approximations.

- the influence of discretization of the integral (13), when using discrete measurement points.

- the influence of the noise present in the input data, corresponding to measurement uncertainties. 


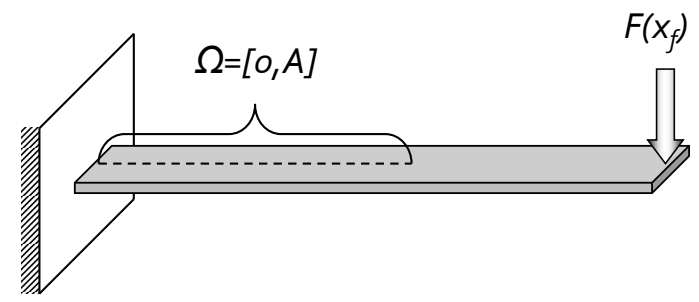

Figure 5: An Euler-Bernoulli cantilever beam, $\Omega$ represents the measurement domain. $x_{f}>A$

The aim of the present section is to identify the influence of the truncation number $N$ on identification, in the absence of errors arising from discretization of the integral, or noise on the measured displacements.

The displacements are computed analytically using Maple software, according to expression (4) for the case of the cantilever beam shown in Fig. 5. The integral of expression (13) is also computed analytically. Fig.6 shows the estimated shear force at the boundary $x=0$, computed using three different Taylor series expansions $(N=3,5$ and 7$)$, expressed as a function of the number of wavelengths in the integration domain. Fig.7 shows (on a logarithmic scale) the corresponding relative identification error, given by the ratio between the identified and exact values.

It should be noted that the same representation is used, whatever the frequency (see [20]). By comparing Fig.4 with Fig.7, it can be seen that the identification error is directly related to the error in the truncated Taylor series expansion. The identification is correct for $A<\lambda / 4$ when $N=3$, for $A<3 \lambda / 8$ when $N=5$, and for $A<\lambda / 2$ when $N=7$ ( $A$ being the size of the integration domain $\Omega$ ). These values are similar to those observed in Fig. 4 and appear to be appropriate limits for the selection of the order of the polynomial test function $P_{N}(x)$. Although a more accurate estimation is achieved for greater values of $N$, when the analytical expression is used in the absence of noise, it is shown in the following section that the estimation's robustness with respect to noise follows a different pattern.

It should be noted that neither the structural parameters of the beam, 


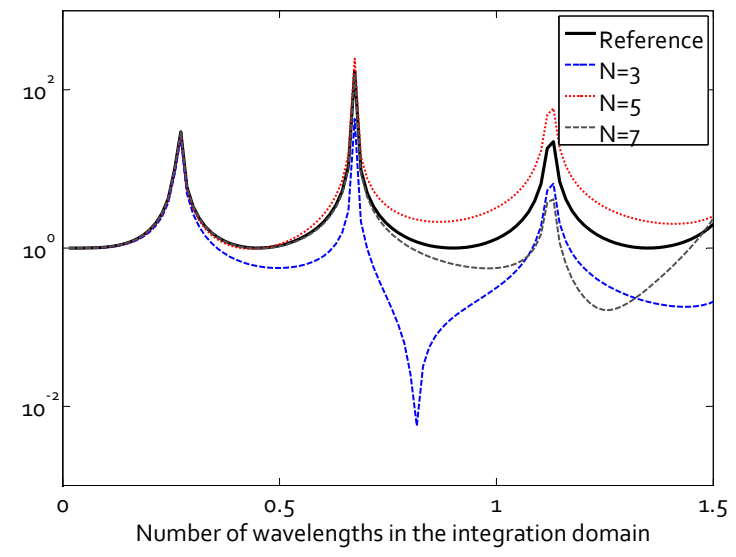

Figure 6: Boundary shear force estimation (analytical computations)

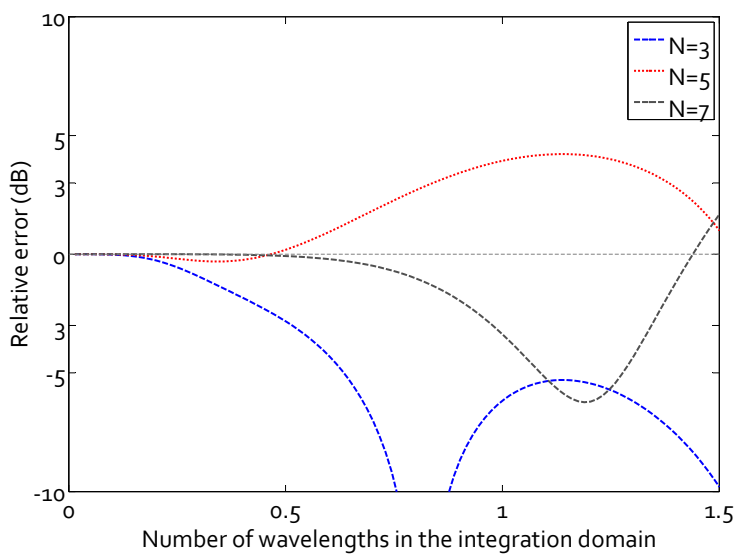

Figure 7: Relative error in the boundary shear force estimation (analytical computations)

nor the size of the domain $\Omega$, change the behaviour of these errors. The parameters selected for the simulation and used here as reference values are: Steel beam, length: $1 \mathrm{~m}$, width: $5 \mathrm{~cm}$, height: $1 \mathrm{~cm}, A=0.95 \mathrm{~m}$. 


\section{Numerical Simulations}

In practice, displacements must be measured at distinct locations, and the integral (13) must be discretized. This is one of the approximations associated with the present method, even when theoretically exact displacements are used. When the displacements are measured, the measurement uncertainties introduce additional errors into the identification.

\subsection{Numerical integration}

Various numerical techniques can be used to discretize an integral. In [20] or [21], the trapezoidal method was studied for similar problems, but was found to be highly sensitive to measurement noise. This technique thus requires too many measurement points to be of any practical interest in the case of the present study. The Gauss-Legendre method is known to be exact when integrating polynomial functions if the discretization uses a sufficiently large number of points. In the general case, the integral approximation can be written:

$$
\int_{0}^{A} f(x) d x \simeq A / 2 \sum_{i=1}^{n} W_{i} f\left(x_{i}\right)
$$

where $f(x)$ is the function to be integrated, $n$ is the number of points used, and $W_{i}$ are the weights applied to each of these points $x_{i}$. In practice, the Gauss-Legendre method has the advantage of producing a better approximation, but requires the implementation of an accurate, irregular mesh. Moreover, this method does not necessitate the knowledge of the function's value at the boundaries. This can be an advantage when it is not possible to make measurements at the boundaries of the beam. It is known that an n-point Gaussian quadrature integral yields an exact result for polynomials of degree $2 n-1$ or less. This approach appears to be naturally more suitable for the analysis of the present problem. In fact, $N$ is the truncation parameter of the Taylor expansion, but is also the degree of the weight function $P_{N}(x)$. Consequently, Eq.(13) can be thought of as the integral of a polynomial function of degree $2 N$. The Gauss-Legendre integration is used in the analyses presented in the following.

\subsection{Influence of noise}

The aim of this section is to demonstrate the influence of noisy displacements (which can be expected when using experimental data) on the reconstructed shear force, and to show that this method is not characterized by the usual instabilities of inverse problems. The case studied here is based on that used in the previous section (Fig. 5), but noise has been added to 
the input data. In order to take these errors into account, and to illustrate their influence on the method, the integral (13) is computed using noisy displacements. The displacement noise is of the form:

$$
v_{N}^{\text {noisy }}\left(x_{i}\right)=v_{N}^{\text {exact }}\left(x_{i}\right) \cdot \Delta v_{m} \cdot e^{j \Delta \phi}
$$

where $\Delta v_{m}$ is a real random number (with a Gaussian distribution) having a mean value equal to unity and a standard deviation equal to $1 / 100 \mathrm{x}$ the magnitude of the displacement. $\Delta \phi$ is another Gaussian real random number having a zero mean value and a standard deviation equal to $1 \mathrm{deg}$. This type of noise is representative of the measurement accuracy. The simulations use only 8 Gaussian integration points. With this number of points, the Gauss-Legendre integration is theoretically exact for the integration of a 15 degree polynomial function. This number was chosen with respect to Eq.(13). The highest degree polynomial test function presented in this study is $N=7$, also implying the use of a $7^{\text {th }}$ degree Taylor expansion, and leading to a globally $14^{\text {th }}$ degree function to be integrated. As a consequence, the errors are not directly related to a problem of discretization. Fig. 8 shows the shear forces at the boundary $x=0$, estimated using noisy

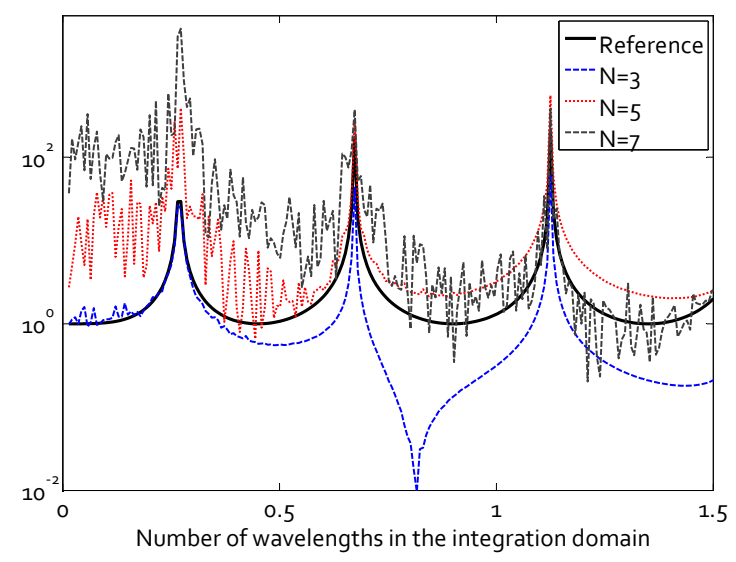

Figure 8: Estimation of the boundary shear force, using various truncation numbers and their corresponding test functions (noisy data)

displacements for three different Taylor series expansions $(N=3,5$ and 7 ), as a function of the number of wavelengths in the integration domain. Fig.9 shows, on a logarithmic scale, the resulting relative error of the identification. These figures show that for a small number of wavelengths in the integration domain, the method becomes more sensitive for greater values of $N$. In the case of a small value of $N(N=3)$, the polynomial function 


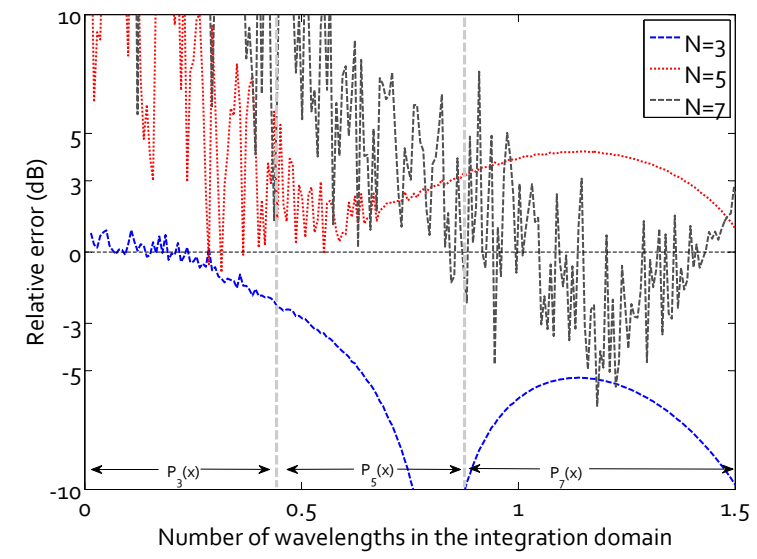

Figure 9: Relative error $(\mathrm{dB})$ on the boundary shear force estimation, using various truncation numbers and their corresponding test functions (noisy data).

$P_{3}(x)$ is smooth (see annex) and acts like a low pass filter, thereby reducing the influence of noise. When $N$ increases, the polynomial's natural ability to filter noise appears to be shifted towards slightly higher wave numbers.

As a consequence, the choice of the correct parameter $N$ and its associated test function $P_{N}(x)$ results from a compromise between a good Taylor series approximation and an adequate filtering capability. In Fig. 9 , the vertical dotted lines (set at 0.4 and 0.9 wavelengths) provide an approximate indication of the regions in which it is recommended to use each of the three polynomial functions, in order to obtain a global error below $3 d B$. These choices were applied to the reconstruction of shear forces in the cantilever beam, using each of the 3 polynomial functions over various ranges. As the limits are not accurately defined, basic weight functions were designed, as shown in Fig. 10. These weights were applied directly to the spectrum of Fig. 8, to reconstruct the new estimation shown in Fig. 11. The simulation is accurate over the observed range of frequencies. It should be noted that for a higher number of wavelengths, the estimation is necessarily inaccurate, since the method is based on the fact that the displacement field can be approximated by a limited Taylor series expansion. 


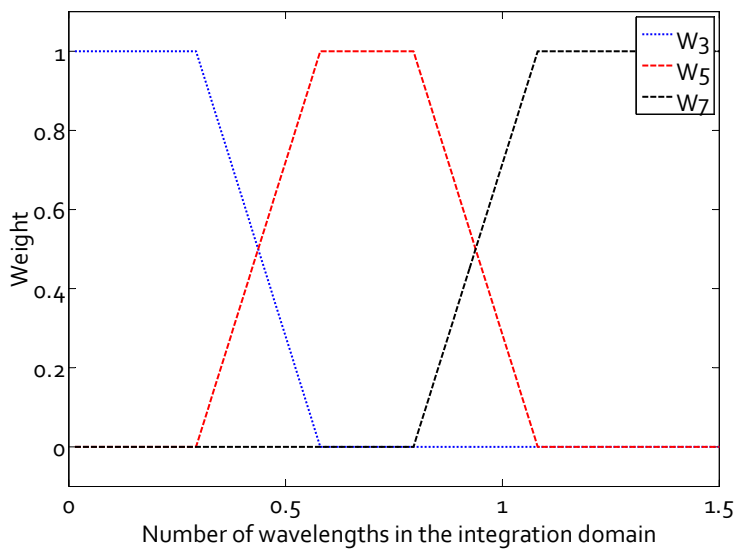

Figure 10: Weight functions: $W 3, W 5$ and $W 7$

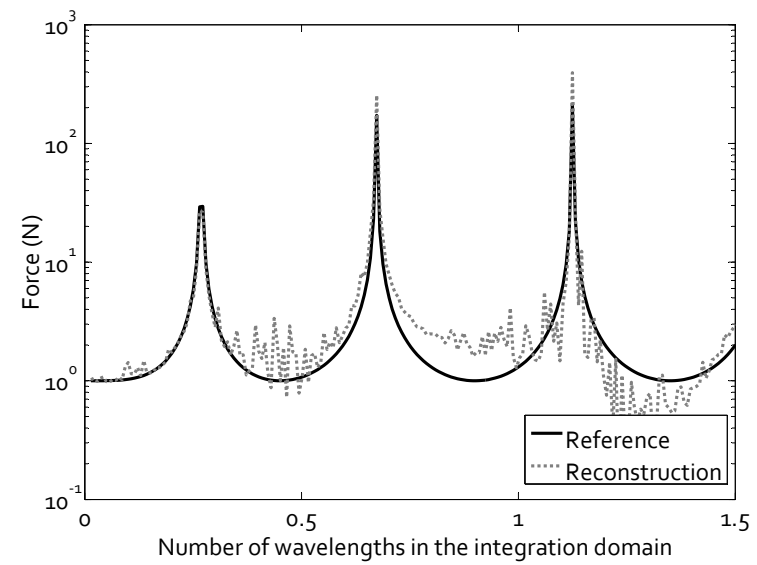

Figure 11: Boundary shear force estimation using various truncation numbers and their corresponding test functions (noisy data), following application of the weight functions.

\section{Experimental validation}

\subsection{Experimental setup}

Various experiments were carried out, in order to validate the proposed method with measured displacements, in the case of shear force identifications. A Gauss-Legendre integration was implemented, using 8 points in 


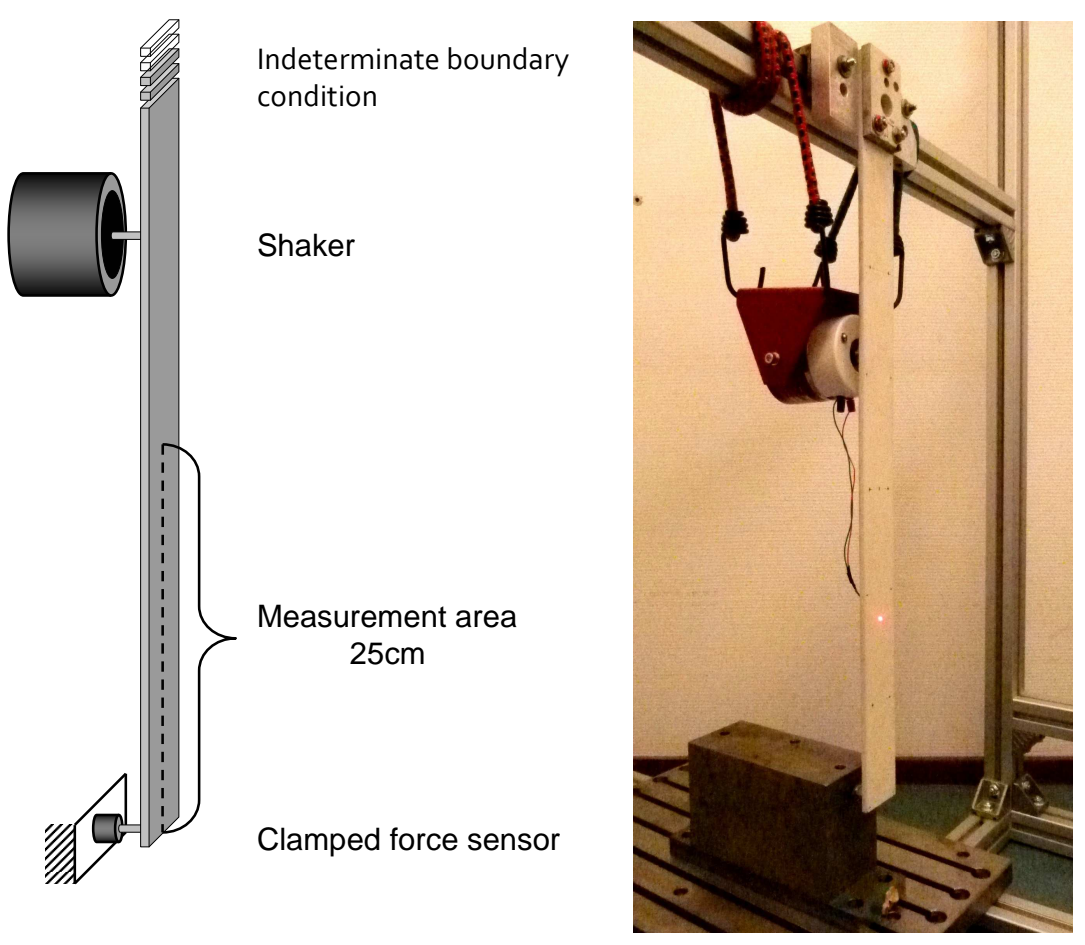

Figure 12: Experimental setup

a $25 \mathrm{~cm}$ domain. The beam was aligned vertically, attached at its upper end, and excited by a shaker at a point outside the measurement area (see Fig. 12). All displacements were measured with a laser vibrometer and random band excitation was used to study the reconstruction over a wide range of frequencies. As the method is local, the quality of the boundary fixation and location of the shaker (outside the measured domain $\Omega$ ) had no influence on the results. A force sensor was placed at the lower boundary, between the beam and a rigid support. This measurement, corresponding to the identified boundary shear force, was used as a reference. The beam was made of steel, and its dimensions were: width $l=5 \mathrm{~cm}$, height $h=0.5 \mathrm{~cm}$.

\subsection{Results}

\subsubsection{Displacement field measurement and design of the weight function $\mathrm{W}$}

In order to estimate the forces over a large range of frequencies, the first step involves defining frequency limits and appropriate weight functions W. Simulations showed that these limits could be set at approximately 
0.4 NoWID and 0.9 NoWID (see Fig. 10). These limits can be found in terms of frequencies when looking at the shape of the displacement fields. At $190 \mathrm{~Hz}$ and $1100 \mathrm{~Hz}$ (see Figs.13 and 14) the displacement fields using 8 Gauss points correspond to these criteria.

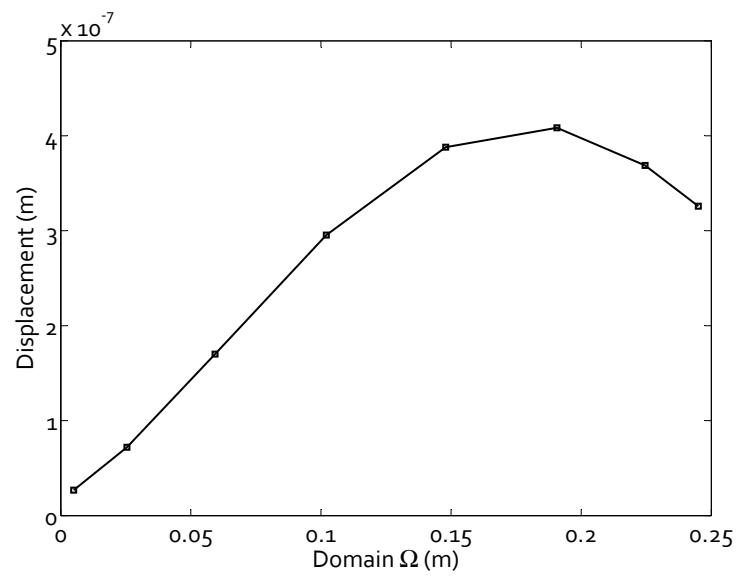

Figure 13: Measured displacements at 8 Gauss points in the domain $\Omega$, at $190 \mathrm{~Hz}$

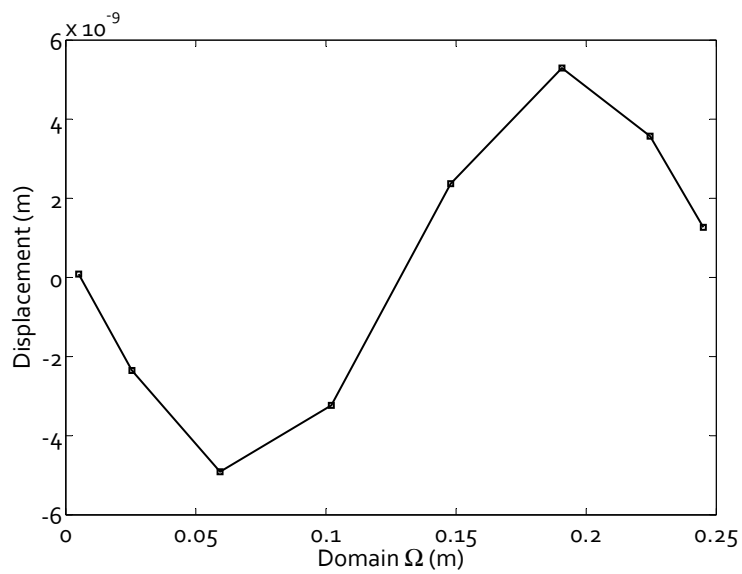

Figure 14: Measured displacements at 8 Gauss points in the domain $\Omega$, at $1100 \mathrm{~Hz}$

In practice, these limits are not exact, and can vary slightly. Due to the proximity of the boundary, the analytical wavelength (Eq.5) is not repre-

Published in Journal of Sound and Vibration, Vol. 333(24), 2014, p6438-6452 
sentative of the observed shapes, and this parameter had to be chosen by observing the displacement field as done previously. The cutoff frequencies of the weight functions $\mathrm{W}$ were simply computed by adding or removing $10 \%$ of the chosen frequency limits. These weight functions are equal to 1 in the frequency range of interest. At the cutoff frequency, they linearly decrease until zero, and are equals to 0.5 when crossing the chosen frequency limits identified in Fig.13 and 14. Fig. 15 shows these 3 weight functions.

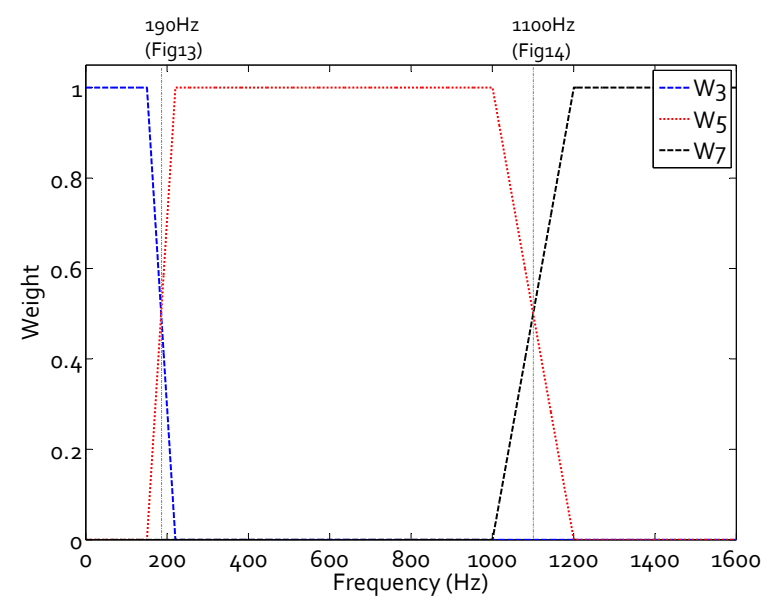

Figure 15: Weight functions $W 3(f), W 5(f)$ and $W 7(f)$

\subsubsection{Amplitude and phase reconstruction using 8 Gauss inte- gration points}

Figs. 16 and 17 show the amplitude and phase of the reconstructed shear forces, which are compared with the reference measurements. As predicted by the simulations, the amplitude and phase are very close to the values given by the reference sensor over the observed range of frequencies. The estimations given by independent test functions can be found in the annex. The small differences observed in Figs. 16 and 17 can be explained by the fact that the reference sensor does not only measure the shear force from the Euler Bernoulli theory. Indeed, due to the presence of this sensor, a point force is created at the location where the Saint Venants principle is not locally respected. Differences also appear at frequencies for which the test function is shifted. These are due mainly to a loss of phase information in the estimated complex frequency response function. 


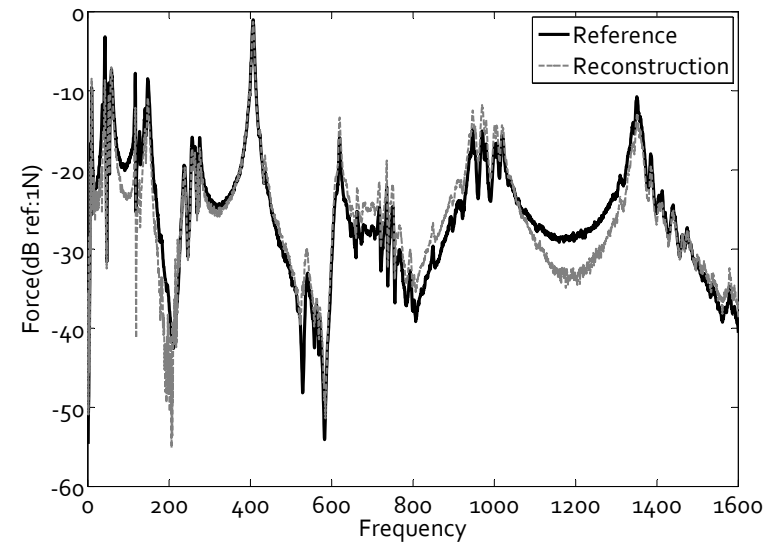

Figure 16: Amplitude of the reference force sensor and force reconstruction, using 8 measurement points and 3 polynomial test functions with their associated weights.

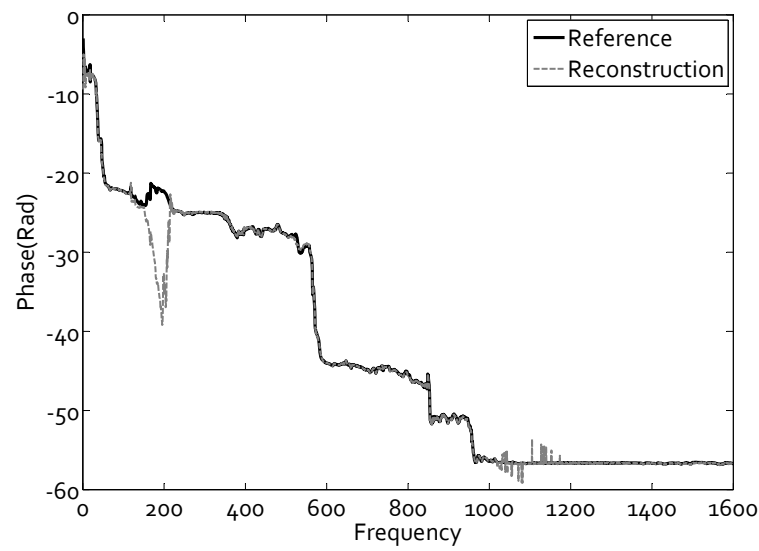

Figure 17: Phase of the reference force sensor and force reconstruction, using 8 measurement points and 3 polynomial test functions with their associated weights.

\section{Conclusion and discussions}

This paper shows that the identification of boundary forces can be understood in terms of the determination of boundary spatial derivatives of transverse displacements. It is proposed to approximate the displacements 
in the vicinity of the boundary by Taylor series expansions, thus allowing the spatial derivatives to be estimated using specific point-wise derivative estimators. This approach makes it possible to extract these quantities through the use of a weighted spatial integration of the displacement field. Numerical simulations using exact and noisy data have revealed the relationship between the size of the integration domain, wavelength, and noise sensitivity, allowing an appropriate truncation order to be selected. Experimental measurements have demonstrated the feasibility and accuracy of the proposed method.

In comparison with others force identification methods, the proposed approach presents some drawbacks. The use of Gauss points to discretize and to estimate integral (13) can appear as a difficult task. The location of each measurement point has to be as accurate as possible. Depending on the measurement set up, it can be time consuming. But, nowadays, the use of technologies like scanning vibrometer makes this part easy and fast. The point coordinates are automatically generated and the measurement points are very accurately located. Nevertheless the different points are not measured simultaneously and real time estimation of the boundary forces is not allowed with this technology. The main limitation of the proposed approach is the fact that frequency range is directly linked to the size of the measurement domain and the truncation order used for the Taylor expansion estimation. Compared to numerical model-based methods as [4], the main advantage of the proposed approach is its local aspect. No global measurement is needed, only a few number of point near the boundary is required. The only restriction is that the equation of motion (2) (leading to eqs (3)) has to be verified inside the measurement domain $\Omega$. Another advantage is that there is no particular equation to solve or a regularization step to tune after the measurement step (contrary to [16] or [18]). Some simple weights have to be applied at each measurement points to obtain directly an estimation of the boundary forces. We remind that these weights are associated to Gauss points or to the weight functions W linked to the truncation order used for the Taylor expansion estimation. From this standpoint the approach is simple to apply. 


\section{Bibliography}

\section{References}

[1] G. Pavic. Measurement of structure borne wave intensity, part i: Formulation of the methods. Journal of Sound and Vibration, 49(2):221230, 1976.

[2] D.U. Noiseux. Measurement of power flow in uniform beams and plates. J. Acoust. Soc. Am., 47:238-247, 1970.

[3] Q. Leclere, C. Pezerat, B. Laulagnet, and L. Polac. Indirect measurement of main bearing loads in an operating diesel engine. Journal of Sound and Vibration, 286(1):341 - 361, 2005.

[4] E. Parloo, P. Verboven, P. Guillaume, and M. Van Overmeire. Force identification by means of in-operation modal models. Journal of Sound and Vibration, 262(1):161-173, 2003.

[5] K. Maes, E. Peeters, J. and. Reynders, G. Lombaert, and G. De Roeck. Identification of axial forces in beam members by local vibration measurements. Journal of Sound and Vibration, 332(21):5417 - 5432, 2013.

[6] C. Pezerat and J.-L. Guyader. Two inverse methods for localisation of external source exciting a beam. Acta Acust, 1(3):1-10, 1995.

[7] Y. Zhang and J.III Adin Mann. Measuring the structural intensity and force distribution in plates. J. Acoust. Soc. Am., 99(1):345-361, 1999.

[8] C. Pezerat and J.-L. Guyader. Force analysis technique: Reconstruction of force distribution on plates. Acta Acust, 86:322-332, 2000.

[9] Q. Leclère and C. Pézerat. Vibration source identification using corrected finite difference schemes. Journal of Sound and Vibration, 331(6):1366-1377, 2012.

[10] C. Renzi, C. Pezerat, and J.-L. Guyader. Vibratory source identification by using the finite element model of a subdomain of a flexural beam. Journal of Sound and Vibration, 332(3):545-562, 2013.

[11] J. Sanchez and H. Benaroya. Review of force reconstruction techniques. Journal of Sound and Vibration, 333(14):2999 - 3018, 2014.

[12] Y.E. Lage, N.M.M. Maia, M.M. Neves, and A.M.R. Ribeiro. Force identification using the concept of displacement transmissibility. Journal of Sound and Vibration, 332(7):1674 - 1686, 2013. 
[13] M.I. Mottershead, J.E.and Friswell. Model updating in structural dynamics: A survey. Journal of Sound and Vibration, 167(2):347 - 375, 1993.

[14] H. Ahmadian, J.E. Mottershead, and M.I. Friswell. Regularisation methods for finite element model updating. Mechanical Systems and Signal Processing, 12(1):47 - 64, 1998.

[15] M.I. Friswell, J.E. Mottershead, and H. Ahmadian. Combining subset section and parameter constraints in model updating. Journal of Vibration and Acoustics, 120:854-859, 1998.

[16] L. Majkut. Identification of beams boundary conditions in ill-posed problem from measured displacements. Journal of theoretical and applied mechanics, 44:91-105, 1993.

[17] R. Ghandhi. Structural optimisation with frequency constraint: a review. American Institute of Aeronautics and Astronautics Journal, 31:296-3203, 1993.

[18] H. Ahmadian, J.E. Mottershead, and M.I. Friswell. Boundary condition identification by solving characteristic equations. Journal of Sound and Vibration, 247(5):755 - 763, 2001.

[19] L. Gavric and G. Pavic. Finite element method for computation of structural intensity by normal mode approach. Journal of Sound and Vibration, 164(1):29-43, 1993.

[20] S. Chesne, C. Pezerat, and J.-L. Guyader. Identification of boundary forces in beams from measured displacements. Journal Vibration Acoustics , ASME transaction, 128:757-771, 2006.

[21] S. Chesne, C. Pezerat, and J.-L. Guyader. Identification of plate boundary forces from measured displacements. Journal Vibration Acoustics , ASME transaction, 130:041006-1, 2008.

[22] S. Chesne and C. Pezerat. Distributed piezoelectric sensors for boundary force measurements in euler bernoulli beams. Smart Mater Struct, 20:9, 2011.

[23] M. Mboup, C. Join, and M. Fliess. Numerical differentiation with annihilators in noisy environment. Numerical Algorithm, 50(4):439467, 2009.

[24] C. Chochol, S. Chesne, and D. Remond. An original differentiation tool for identification on continuous structures. Journal of Sound and Vibration, 332(13):3338 - 3350, 2013. 
[25] M. Fliess and H. Sira-Ramirez. An algebraic framework for linear identification. ESAIM: Control, Optimisation and Calculus of Variations, 9:151-168, 82003.

[26] M. Fliess, S. Fuchshumer, M. Schöberl, K. Schlacher, and H. SiraRamirez. An introduction to algebraic discrete-time linear parametric identification with a concrete application. Journal Européen des Systèmes Automatisés, 42(2-3):210-232, 2008.

[27] C. Lanczos. Applied Analysis. Dover Books on Mathematics. Dover Publications, 1988.

[28] S.K. Rangarajan and Purushothaman S.P. Lanczos generalized derivative for higher orders. Journal of Computational and Applied Mathematics, 177(2):461 - 465, 2005.

[29] J.-L. Guyader. Vibration in Continuous Media. Wiley-Blackwell, 2006.

\section{A Polynomial functions}

In this annex the expression $P_{N}(x)$ is derived, for the estimation of the third derivative over a domain $\Omega=[0, A]$. These polynomial functions are shown in Fig. A.1 (for $A=1$ ).

$$
\begin{gathered}
P_{3}(x)=-7 ! /\left(6 A^{7}\right) \times\left[(A-x)^{3}-9(A-x)^{2} x+9(A-x) x^{2}-x^{3}\right] \quad(\mathrm{A} .1) \\
P_{5}(x)=-9 ! /\left(12 A^{9}\right) \times \\
{\left[3 / 2(A-x)^{5}-69 / 2(A-x)^{4} x+\right.} \\
\left.127(A-x)^{3} x^{2}-117(A-x)^{2} x^{3}+27(A-x) x^{4}-x^{5}\right] \\
P_{7}(x)=-11 ! /\left(144 A^{11}\right) \times \\
{\left[2(A-x)^{7}-434 / 5(A-x)^{6} x+3486 / 5(A-x)^{5} x^{2}-(\mathrm{A} .3)\right.} \\
\left.1742(A-x)^{4} x^{3}+1579(A-x)^{3} x^{4}-519(A-x)^{2} x^{5}+53(A-x) x^{6}-x^{7}\right]
\end{gathered}
$$

\section{B Shear force estimation with various test func- tions}

Fig. B.2 shows the amplitude spectrum measured by the reference force sensor, as well as the estimated boundary shear force spectrum, computed 


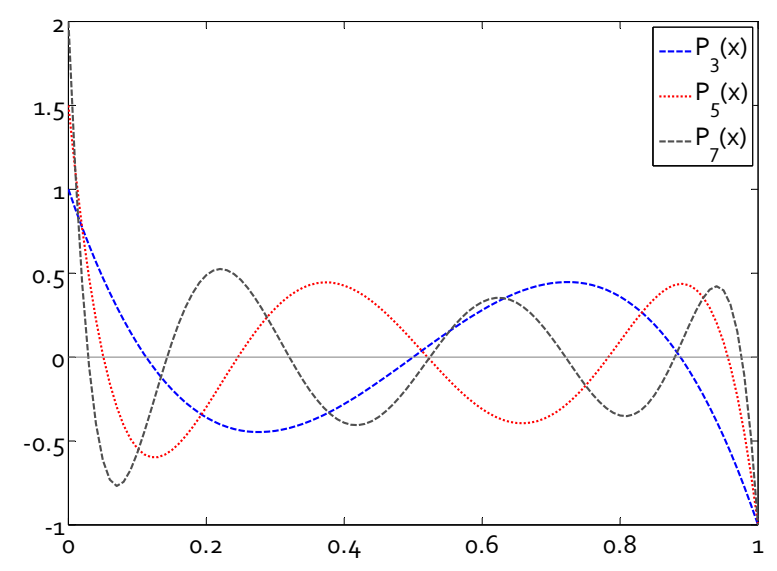

Figure A.1: Polynomial test functions $P_{3}(x), P_{5}(x)$ and $P_{7}(x)$

using various test functions without weights. The measured behaviour can be seen to be very similar to that determined by simulation. At low frequencies, a high value of $N$ leads to a greater sensitivity to noise. As the frequency increases, the term $N$ at which the Taylor series expansion is truncated also needs to be increased, in order to maintain the accuracy of the estimation.

\section{Amplitude reconstruction using 15 Gauss inte- gration points}

In order to test the proposed method, an additional experiment was carried out using 15 Gauss integration points. With this number of points, improved reconstruction quality could be expected. Fig. C.3 shows the amplitude spectra given by the reference force sensor, together with the boundary shear force estimations determined using 15 integration points. The same truncation numbers $N$ and the same weight function $W$ were used. The reconstruction is plotted over a larger frequency range ([0-3200] $\mathrm{Hz}$, rather than $[0-1600] \mathrm{Hz})$. At approximately $1800 \mathrm{~Hz}$, the number of wavelengths included in the integration domain $\Omega$ exceeds 1.5. At this frequency, the Taylor series approximation is no longer valid and the estimation would theoretically be incorrect. The spectra shown in Fig. C.3 confirm this theoretical consideration, as well as the observations done in the simulations.

In order to improve the results at such frequencies, the value of $N$ and the number of measurement points should be increased. This modification 


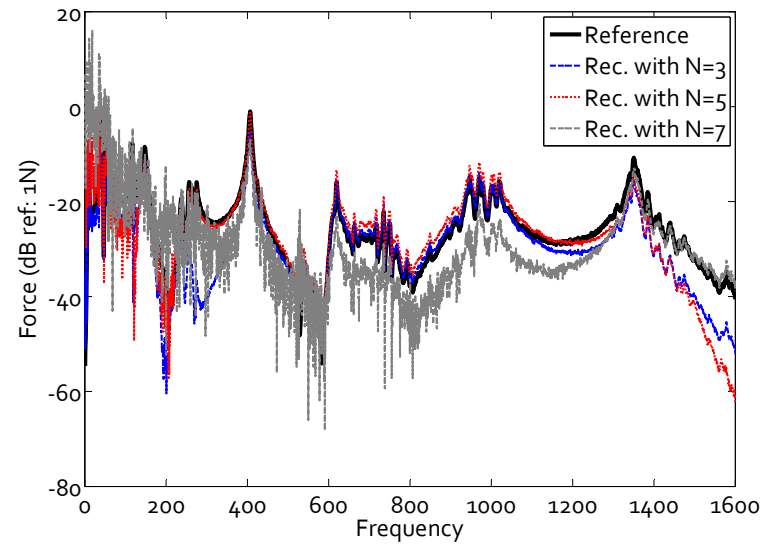

Figure B.2: Amplitude spectra corresponding to the reference force sensor measurements, and the simulated force reconstruction, using 8 measurement points and the test functions $P_{3}, P_{5}$ and $P_{7}$

\begin{tabular}{l|c|c|c|c|c|c|c|c} 
Point & $x_{1}$ & $x_{2}$ & $x_{3}$ & $x_{4}$ & $x_{5}$ & $x_{6}$ & $x_{7}$ & $x_{8}$ \\
\hline \hline Location $(\mathrm{cm})$ & 0.0050 & 0.0254 & 0.0593 & 0.1021 & 0.1479 & 0.1907 & 0.2246 & 0.2450 \\
Weight & 0.0127 & 0.0278 & 0.0392 & 0.0453 & 0.0453 & 0.0392 & 0.0278 & 0.0127
\end{tabular}

Table 1: Locations and the weights of the Gauss points for $N=8$ on a 25 $\mathrm{cm}$ length domain

should however be accompanied by measurements characterised by a very low level of noise, since a higher truncation number implies an increase in noise sensitivity.

\section{Gauss points locations and weight}

The tables 1 and 2 give the locations and the weights of the Gauss points for $N=8$ and $N=25$, on a $25 \mathrm{~cm}$ length domain. 


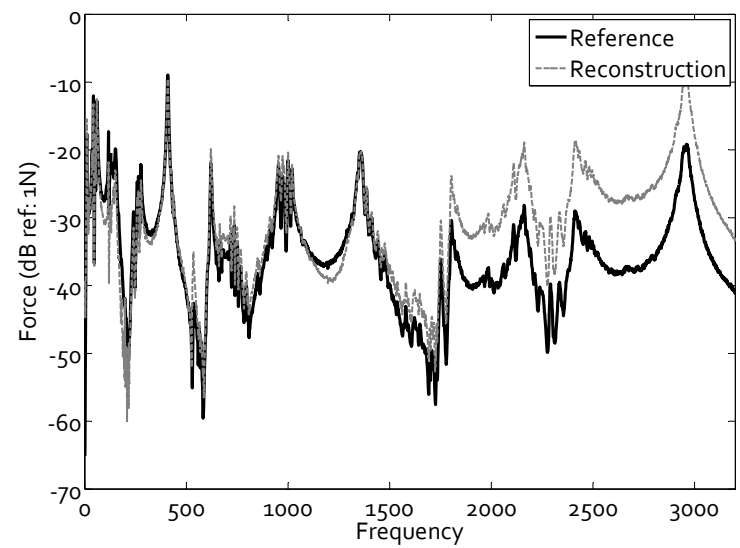

Figure C.3: Amplitude spectra corresponding to the reference force sensor and the force reconstruction simulations, made using 15 measurement points and 3 polynomial test functions, associated with weights applied to the high frequency range.

\begin{tabular}{l|c|c|c|c|c|c|c|c} 
Point & $x_{1}$ & $x_{2}$ & $x_{3}$ & $x_{4}$ & $x_{5}$ & $x_{6}$ & $x_{7}$ & $x_{8}$ \\
\hline \hline Location(cm) & 0.0015 & 0.0078 & 0.0190 & 0.0344 & 0.0536 & 0.0757 & 0.0999 & 0.1250 \\
Weight & 0.0038 & 0.0088 & 0.0134 & 0.0174 & 0.0208 & 0.0233 & 0.0248 & 0.0253 \\
\hline & & & & & & & & \\
Point & $x_{9}$ & $x_{10}$ & $x_{11}$ & $x_{12}$ & $x_{13}$ & $x_{14}$ & $x_{15}$ & \\
\hline \hline Location $(\mathrm{cm})$ & 0.1501 & 0.1743 & 0.1964 & 0.2156 & 0.2310 & 0.2422 & 0.2485 & \\
Weight & 0.0248 & 0.0233 & 0.0208 & 0.0174 & 0.0134 & 0.0088 & 0.0038 &
\end{tabular}

Table 2: Locations and the weights of the Gauss points for $N=15$ on a 25 $\mathrm{cm}$ length domain 